\title{
Exotic Heavy Quark Spectroscopy - Theory Interpretation vs Data
}

\section{Christian Hambrock*}

TU Dortmund

E-mail: christian.hambrocketu-dortmund.de

An overview over recent spectroscopy results in comparison with data is given. We focus on the exotica $Z_{b}(10610), Z_{b}(10650)$ and $Y_{b}(10890)$ in the bottom sector and discuss a possible connection to the exotica in the charm sector, namely to the resonances $Y_{c}(4260)$ and $Z_{c}(3900)$. Furthermore, future prospects for the determination of the nature of $Y_{b}(10890)$ are outlined.

14th International Conference on B-Physics at Hadron Machines April 8-12, 2013

Bologna, Italy

\footnotetext{
* Speaker.
} 


\section{Introduction}

The term exotica labels states which have an identical number of quarks and antiquarks, but defy an ordinary meson classification. Many exotic states in the charm sector with $c \bar{c}$ content have been discovered by Belle and others, see $[1,2]$ and references therein. While there are most likely many more which are yet unknown, all of them should also reflect in the $b \bar{b}$ sector, according to heavy quark symmetry. The non-discovery of the respective $b \bar{b}$ partners of the charmonium-like exotica would be even more enigmatic. Indeed, there exist three candidates up to date, namely the states labeled $Y_{b}(10890), Z_{b}(10610)$ and $Z_{b}(10650)$, observed by Belle [3]. The scarceness of the discovered $b \bar{b}$ states, compared to the charm ones, is mostly owed to the current status of experimental data; the charm sector can be probed by the $e^{+} e^{-}$machines not only directly, but also through $B$ meson decays and initial state radiation. The bottom sector on the other hand is relying on direct measurements, most of which have been tuned to the center-of-mass energy of the masses of $\Upsilon(4 S)$ and $\Upsilon(5 S)$. The dataset for the latter has roughly one order of magnitude smaller statistics than the former. Notably, all three exotic $b \bar{b}$ candidates have firstly been discovered in that sample. This is not surprising, since exotica lie generically above the open heavy quark thresholds due to their multiquark nature. A list of the observed exotica is given in Table 1.

Models to accommodate the exotica have been proposed over the last decades. The molecular interpretation (dimeson states bound similar to nuclei) [4, 5, 6] is favored for some states, while the tetraquark interpretation (four quark states which are genuinely bound by gluons) $[7,8,9]$ is favored for others. Hybrids [10] (quarks plus glueballs) play a slightly minor role in the current discussion, but are also studied in this context. However, a consistent picture is missing. None of the present models can explain all states simultaneously, while in addition more predicted states are missing than are discovered in either ansatz. The status in the charm sector is sketched in Figure 1. For the molecular interpretation, it is not clear, which combination of mesons should bind; there is currently no deeper understanding, why some states are observed (like for example the $X_{c}(3872)$,

Table 1: Exotic states found by Belle and others $[1,2]$. An asterisks indicates the first discovery before Belle.

\begin{tabular}{|c|c|c|c|c|c|c|c|}
\hline State & $M(\mathrm{MeV})$ & $\Gamma(\mathrm{MeV})$ & $J^{P C}$ & Decay Modes & Production Modes & Also observed by & date \\
\hline$Y_{S}(2175)$ & $2175 \pm 8$ & $58 \pm 26$ & $1^{--}$ & $\begin{array}{l}\phi f_{0}(980) \\
\pi^{+} \pi^{-} J / \psi\end{array}$ & $\begin{array}{l}e^{+} e^{-}(\text {ISR) } \\
J / \psi \rightarrow \eta Y_{S}(2175)\end{array}$ & $\begin{array}{l}\text { BaBar*, BESII } \\
\text { BaBar }\end{array}$ & 2006 \\
\hline$X(3872)$ & $3871.4 \pm 0.6$ & $<2.3$ & $1^{++}$ & $\gamma J / \psi, D D^{-*}$ & $B \rightarrow K X(3872), p \bar{p}$ & CDF, D0 & 2003 \\
\hline$Z(3900)$ & $3899 \pm 6$ & $46 \pm 22$ & $1^{+}$ & $\pi^{ \pm} J / \psi$ & $Z(4260) \rightarrow Z(3900) \pi$ & BESIII* $^{*}$ & 2013 \\
\hline$X(3915)$ & $3914 \pm 4$ & $28_{-14}^{+12}$ & $0 / 2^{++}$ & $\omega J / \psi$ & $\gamma \gamma \rightarrow X(3915)$ & & 2009 \\
\hline$Z(3930)$ & $3929 \pm 5$ & $29 \pm 10$ & $2^{++}$ & $\begin{array}{l}D \bar{D} \\
D \bar{D}^{*}(\text { not } D \bar{D}\end{array}$ & $\gamma \gamma \rightarrow Z(3940)$ & & 2009 \\
\hline$X(3940)$ & $3942 \pm 9$ & $37 \pm 17$ & $0^{?+}$ & or $\omega J / \psi)$ & $e^{+} e^{-} \rightarrow J / \psi X(3940)$ & & 2005 \\
\hline$Y(3940)$ & $3943 \pm 17$ & $87 \pm 34$ & $?^{?+}$ & $\omega J / \psi\left(\operatorname{not} D \overline{D^{*}}\right)$ & $B \rightarrow K Y(3940)$ & BaBar & 2005 \\
\hline$Y(4008)$ & $4008_{-49}^{+82}$ & $226_{-80}^{+97}$ & $1^{--}$ & $\pi^{+} \pi^{-} J / \psi$ & $e^{+} e^{-}(\mathrm{ISR})$ & & 2005 \\
\hline$X(4160)$ & $4156 \pm 29$ & $139_{-65}^{+113}$ & $0^{?+}$ & $D^{*} \bar{D}^{*}(\operatorname{not} D \bar{D})$ & $e^{+} e^{-} \rightarrow J / \psi X(4160)$ & & 2008 \\
\hline$Y(4260)$ & $4264 \pm 12$ & $83 \pm 22$ & $1^{--}$ & $\pi^{+} \pi^{-} J / \psi$ & $e^{+} e^{-}$(ISR) & $\mathrm{BaBar}^{*}$, CLEO & 2005 \\
\hline$Y(4350)$ & $4361 \pm 13$ & $74 \pm 18$ & $1^{--}$ & $\pi^{+} \pi^{-} \psi^{\prime}$ & $e^{+} e^{-}$(ISR) & BaBar* & 2007 \\
\hline$X(4630)$ & $4634_{-11}^{+9}$ & $92_{-32}^{+41}$ & $1^{--}$ & $\Lambda_{c}^{+} \Lambda_{c}^{-}$ & $e^{+} e^{-}$(ISR) & & 2008 \\
\hline$Y(4660)$ & $4664 \pm 12$ & $48 \pm 15$ & $1^{--}$ & $\pi^{+} \pi^{-} \psi^{\prime}$ & $e^{+} e^{-}$(ISR) & & 2007 \\
\hline$Z(4050)$ & $4051_{-23}^{+24}$ & $82_{-29}^{+51}$ & $?$ & $\pi^{ \pm} \chi_{c 1}$ & $B \rightarrow K Z^{ \pm}(4050)$ & & 2008 \\
\hline$Z(4250)$ & $4248_{-45}^{+185}$ & $177_{-72}^{+320}$ & $?$ & $\pi^{ \pm} \chi_{c 1}$ & $B \rightarrow K Z^{ \pm}(4250)$ & & 2008 \\
\hline$Z(4430)$ & $4433 \pm 5$ & $45_{-18}^{+35^{2}}$ & $?$ & $\pi^{ \pm} \psi^{\prime}$ & $B \rightarrow K Z^{ \pm}(4430)$ & & 2007 \\
\hline$Z_{b}(10610)$ & $10,607 \pm 2$ & $18.4 \pm 2.4$ & $1^{+}$ & $\pi^{ \pm} h_{b}(1,2 P), \pi^{ \pm} \Upsilon(1,2,3 S)$ & $Y_{b} / \Upsilon(5 S) \rightarrow Z_{b}(10610) \pi$ & & 2011 \\
\hline$Z_{b}(10650)$ & $10.652 \pm 2$ & $11.5 \pm 2.2$ & $1^{+}$ & $\pi^{ \pm} h_{b}(1,2 P), \pi^{ \pm} \Upsilon(1,2,3 S)$ & $Y_{b} / \Upsilon(5 S) \rightarrow Z_{b}(10650) \pi$ & & 2011 \\
\hline$Y_{b}(10890)$ & $10,890 \pm 3$ & $55 \pm 9$ & $1^{--}$ & $\pi^{+} \pi^{-} \Upsilon(1,2,3 S)$ & $e^{+} e^{-} \rightarrow Y_{b}$ & & 2008 \\
\hline
\end{tabular}




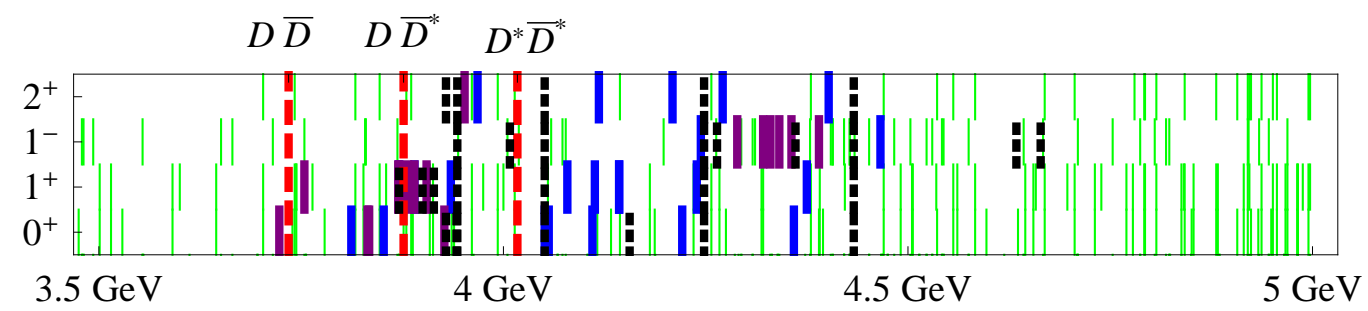

Figure 1: Status of current mass estimates in the charm sector and comparison with data. The data (dashed, black) is shown with corresponding quantum numbers (if unknown, the line stretches over the full range). The theory estimates are given for the molecules as an illustrative naive combination of mesons (thin, green lines), where the masses are taken from the PDG [14]. The tetraquark estimates are taken from [11, 12] based on the relativistic quark model (blue) and from $[7,13]$ based on the constituent quark model (purple).

which is a candidate for a $D^{0} \bar{D}^{0}$ bound state), while a plethora of different possible combinations is absent. On the other hand, the tetraquark picture is incomplete, since reliable calculations for the masses are missing. The calculations in the relativistic quark model [11, 12] are not in good agreement with constituent model estimates $[13,7]$. In addition, neither of the two approaches have good overall agreement with the experiment.

For a theoretical interpretation of the data it is insufficient to rely on the mass estimates only. Hopefully the lattice will provide unbiased input for the masses and fuel the discussion eventually. Phenomenologically, however, one needs to study further characteristics, such as decay patterns. But it is also imperative to find relations among the different states to test an underlying model on a broad basis. The heavy quark symmetry should hold to some extent. We discuss one important puzzle and point out a possible relation between patterns in both heavy quark sectors. It is argued that the states $Y_{c}(4260)$ and $Y_{b}(10890)$, as well as the states $Z_{c}(3900)$ and $Z_{b}(10610,10650)$, might be heavy quark partners.

\section{2. $Y_{b}(10890)$}

The potential exotic state $Y_{b}(10890)$ with $J^{P C}=1^{--}$was first observed by the Belle collaboration $[15,16]$ and remains to be confirmed by independent experiments. The anomalously large production cross sections for $e^{+} e^{-} \rightarrow \Upsilon(1 S, 2 S, 3 S) \pi^{+} \pi^{-}$measured at $\Upsilon(5 S)$ did not agree well with the lineshape and production rates for the conventional $b \bar{b}$ state $\Upsilon(5 S)$. Ordinary $\Upsilon(n S)$ decays are well-described by the multipole expansion [17], which involves coupling to two gluons and are hence Zweig forbidden and do not show any significant resonant structure. Compared to the ordinary $\Upsilon(n S) \rightarrow \Upsilon(m S) \pi^{+} \pi^{-}, m<n$ decays, the derived partial width at $\Upsilon(5 S)$ is out of line by two orders of magnitude. In addition, a distinct resonant structure is observed in the invariant mass spectra. Currently two theoretical explanations are competing; the tetraquark interpretation on the one hand $[18,19]$ and the rescattering model [20] on the other. The former can explain the enhancement and the resonant structure via Zweig allowed decays and coupling to intermediate resonances, while the latter is relying on the decay $\Upsilon(5 S) \rightarrow B^{(*)} \bar{B}^{(*)}$ and a subsequent recombination of the $B$ mesons. 


\section{3. $Z_{b}(10610)$ and $Z_{b}(10650)$}

Belle [3] reported the measurement of the $\pi^{ \pm} \Upsilon(n S)(n=1,2,3)$ and $\pi^{ \pm} h_{b}(m P)(m=1,2)$ invariant mass spectra from the data taken near the peak of the $\Upsilon(5 S)$ resonance in the processes $e^{+} e^{-} \rightarrow \Upsilon(n S) \pi^{+} \pi^{-}$and $e^{+} e^{-} \rightarrow h_{b}(m P) \pi^{+} \pi^{-}$, in which two charged bottomonium-like states $Z_{b}^{ \pm}(10610)$ and $Z_{b}^{ \pm}(10650)$ were discovered. It is not clear if the $Z_{b}$ states are the decay products of $Y_{b}(10890)$ or of $\Upsilon(5 S)$, as the Belle measurements are not equivocal on this point, and the production mechanism, obviously central to theoretical interpretation, remains to be unambiguously confirmed. The angular distribution analysis indicates that the quantum numbers of both $Z_{b}^{ \pm}$and $Z_{b}^{\prime \pm}$ are $I^{G}\left(J^{P}\right)=1^{+}\left(1^{+}\right)$, where $Z_{b}$ and $Z_{b}^{\prime}$ are the lighter and heavier flavor eigenstates, which are identified for small mixing with $Z_{b}(10610)$ and $Z_{b}(10650)$, respectively. Their neutral isospin counterparts with $I_{3}=0$ have $J^{P C}=1^{+-}$. The masses and decay widths averaged over the five different final states are [3]:

$$
\begin{aligned}
& m_{Z_{b}^{ \pm}}=10607.2 \pm 2.0 \mathrm{MeV}, m_{Z_{b}^{\prime \pm}}=10652.2 \pm 1.5 \mathrm{MeV}, \\
& \Gamma_{Z_{b}^{ \pm}}=18.4 \pm 2.4 \mathrm{MeV}, \quad \Gamma_{Z_{b}^{\prime \pm}}=11.5 \pm 2.2 \mathrm{MeV} .
\end{aligned}
$$

Due to the proximity of the $Z_{b}$ and $Z_{b}^{\prime}$ masses with the $B \bar{B}^{*}$ and $B^{*} \bar{B}^{*}$ thresholds [21], it has been proposed that the former could be realized as $S$-wave $B \bar{B}^{*}$ and $B^{*} \bar{B}^{*}$ molecular states, respectively $[22,23,24,25,26,27,28]$. In this scenario, the heavy quark spin structure of the $Z_{b}$ and $Z_{b}^{\prime}$ is expected to mimic that of the corresponding meson pairs, which is given in a non-relativistic notation by

$$
\begin{aligned}
& \left|Z_{b}\right\rangle=\left(0_{b \bar{q}}^{-} \otimes 1_{q \bar{b}}^{-}+1_{q \bar{b}}^{-} \otimes 0_{q \bar{b}}^{-}\right) / \sqrt{2}, \\
& \left|Z_{b}^{\prime}\right\rangle=1_{b \bar{q}}^{-} \otimes 1_{q \bar{b}}^{-},
\end{aligned}
$$

where $0^{-}$and $1^{-}$denote the para and ortho states with negative parity, respectively. One anticipates the mass splitting to follow $\Delta m_{Z_{b}} \equiv m_{Z_{b}^{\prime}}-m_{Z_{b}}=m_{B^{*}}-m_{B} \simeq 46 \mathrm{MeV}$, in neat agreement with the observed value $\Delta m_{Z_{b}}=(45 \pm 2.5) \mathrm{MeV}$ [3]. Moreover, the structure in Eq. (3.2) predicts that $Z_{b}$ and $Z_{b}^{\prime}$ should have the same decay width, which is approximately in agreement with the data.

There is a small caveat, since the masses of the states lie above their respective thresholds by about $2 \mathrm{MeV}$. If consolidated by more precise experiments, this feature may become a serious problem in this approach, as a one-pion exchange potential, which would produce such a bound state, does not support an $S$-wave $B \bar{B}^{*}$ resonance above threshold in an effective field theory [29]. Also, the measured total decay widths appear much too large compared to the naively expected ones for loosely bound states, and this suggests that both $Z_{b}$ states are compact hadrons.

In our previous work [30], we calculated the $Z_{b}$ masses in the tetraquark model based on an effective Hamiltonian approach. The agreement with the experimental masses is not particularly good. However, we showed in [31] that by including meson loop contributions and mixing between the heavier and the lighter $Z_{b}$ states, the measured masses can in principle be obtained in parts of the parameter space (depending on the coupling to the dimeson channels).

A more striking pattern emerges based on the heavy quark spin symmetry. There is an analogous expression to the decomposition in (3.2) for the tetraquark states

$$
\begin{aligned}
\left|Z_{b}\right\rangle & =\left(0_{[b q]} \otimes 1_{[\bar{b} \bar{q}]}-1_{[b q]} \otimes 0_{[\bar{b} \bar{q}]}\right) / \sqrt{2}, \\
\left|Z_{b}^{\prime}\right\rangle & =1_{[b q]} \otimes 1_{[\bar{b} \bar{q}]},
\end{aligned}
$$


Table 2: Branching fractions $(\mathscr{B})$ of $Z_{b}(10610)$ and $Z_{b}(10650)$ assuming that the observed so far channels saturate their decays (Table from [32]).

\begin{tabular}{lcc}
\hline \hline Channel & $\mathscr{B}\left(Z_{b}(10610)\right)[\%]$ & $\mathscr{B}$ of $\left(Z_{b}(10650)\right)[\%]$ \\
\hline \hline$\Upsilon(1 S) \pi^{+}$ & $0.32 \pm 0.09$ & $0.24 \pm 0.07$ \\
$\Upsilon(2 S) \pi^{+}$ & $4.38 \pm 1.21$ & $2.40 \pm 0.63$ \\
$\Upsilon(3 S) \pi^{+}$ & $2.15 \pm 0.56$ & $1.64 \pm 0.40$ \\
$h_{b}(1 P) \pi^{+}$ & $2.81 \pm 1.10$ & $7.43 \pm 2.70$ \\
$h_{b}(2 P) \pi^{+}$ & $2.15 \pm 0.56$ & $14.8 \pm 6.22$ \\
$B^{+} \bar{B}^{* 0}+\bar{B}^{0} B^{*+}$ & $86.0 \pm 3.6$ & - \\
$B^{*+} \bar{B}^{* 0}$ & - & $73.4 \pm 7.0$ \\
\hline \hline
\end{tabular}

where square brackets denote the composite diquarks, see [30] for details. Performing a Fierz transformation, the flavor and spin content in the $b \bar{q} \otimes q \bar{b}$ product space can be made explicit:

$$
\begin{aligned}
& \left|Z_{b}\right\rangle=1_{\bar{b} \bar{q}}^{-} \otimes 1_{q \bar{b}}^{-}, \\
& \left|Z_{b}^{\prime}\right\rangle=\left(1_{b \bar{q}}^{-} \otimes 0_{q \bar{b}}^{-}+0_{b \bar{q}}^{-} \otimes 1_{q \bar{b}}^{-}\right) / \sqrt{2} .
\end{aligned}
$$

The labels $0_{b \bar{q}}$ and $1_{b \bar{q}}$ in Eq. (3.4) can be viewed as $\bar{B}$ and $\bar{B}^{*}$, respectively. Eq. (3.4) shows that the $Z_{b}$ and $Z_{b}^{\prime}$ have similar coupling strengths with different final states. It follows that $Z_{b}$ couples to $B^{*} \bar{B}^{*}$ state while $Z_{b}^{\prime}$ couples to $B \bar{B}^{*}$.

After our prediction of this peculiar decay pattern, the Belle collaboration published new measurements on the $Z_{b}$ decays [32], shown in Table 2. The Belle observation, if confirmed by independent experiments, would suggest a dominant molecular component.

\section{4. $Y_{c}(4260)$}

The state $Y_{c}(4260)$ was first observed by BaBar [33] in the final state $J / \psi \pi^{+} \pi^{-}$. The direct production in $e^{+} e^{-}$annihilation indicates $J^{P C}=1^{--}$quantum numbers. There are some striking similarities between the two exotic states $Y_{b}(10890)$ and $Y_{c}(4260)$. They not only have the same quantum numbers, but are furthermore observed in the identical process $H \pi^{+} \pi^{-}$, in which $H$ labels a quarkonium $Q \bar{Q}$ state with $J^{P C}=1^{--}$, with $Q$ being either a $c$ or a $b$ quark (namely $H$ is a member of the $J / \psi$ or $\Upsilon$ family). A naive mass estimate, starting from our constituent quark model estimate for $Y_{b}(10890)$ [30] is obtained by subtracting twice the difference of the constituent quark masses $m_{b}-m_{c} \approx 3333 \mathrm{MeV}$ :

$$
m_{Y_{b}(10890)} \approx m_{Y_{c}(4260)}+2\left(m_{b}-m_{c}\right) .
$$

The estimate is only off by about $30 \mathrm{MeV}$. However, this feature has to be taken with some caution, since the use of heavy quark symmetry in providing reliable mass estimates in multiquark systems remains to be quantitatively tested.

Due to the apparent similarities between $Y_{c}(4260)$ and $Y_{b}(10890)$, we suggested to search for the partners of the $Z_{b}$ states in the $Y(4260)$ decays via the channel $Y(4260) \rightarrow Z_{c}^{ \pm} \pi^{\mp}$ [30]. 


\section{5. $Z_{c}(3900)$}

Indeed in the proposed decay, BESIII observed a state with identical quantum numbers compared to the $Z_{b}$ states in spring 2013 [2], namely the $Z_{c}(3900)$. Several aspects are, however, peculiar in that observation. Only one state was found, of which the mass is moreover roughly $4 \sigma$ above the corresponding $D \bar{D}^{(*)}$ threshold; a finding which is hard to explain in terms of hadronic molecules. But there are some aspects which facilitate the tetraquark interpretation [34].

\section{Prospects for the bottom sector}

Currently there are pending, unanswered questions concerning the exotic spectroscopy in the heavy quark sectors. One big puzzle, depicted in Figure 2, poses an intriguing mystery. Are the exotic states related? If yes, why do the $Z_{c}$ and $Z_{b}$ states appear to be very different? If not, what is the true relation linking the two heavy quark sectors?

To promote the endeavor of understanding the heavy exotic states, the exploration of the bottom sector is important. Not only new states are waiting to be discovered, but also the existence of $Y_{b}(10890)$ needs to be established or refuted.

To achieve this intermediate goal, several opportunities arise, which are outlined in the following, one of which has been proposed in [19]. Based on the tetraquark model, we developed the formalism for the processes

$$
e^{+}+e^{-} \rightarrow Y_{b} \rightarrow \Upsilon(1 S)+P+P^{\prime},
$$

where $P P^{\prime}$ stands for the pseudoscalar-meson pairs $\pi^{+} \pi^{-}, K^{+} K^{-}$and $\eta \pi^{0}$. With this formalism, we analyzed the invariant-mass $M_{P P^{\prime}}$ and the $\cos \theta$ spectra, where $\theta$ is the angle between the momenta of $Y_{b}$ and $P$ in the $P P^{\prime}$ rest frame. The resulting correlations among $\sigma_{\Upsilon(1 S) \pi^{+} \pi^{-}}, \sigma_{\Upsilon(1 S) K^{+} K^{-}}$ and $\sigma_{\Upsilon(1 S) \eta \pi^{0}}$ are worked out. Constraining these correlations using the existing data on the first two processes, we predict $\sigma_{\Upsilon(1 S) \eta \pi^{0}} / \sigma_{\Upsilon(1 S) \pi^{+} \pi^{-}}$. We also predict $\sigma_{\Upsilon(1 S) K^{+} K^{-}} / \sigma_{\Upsilon(1 S) K^{0} \bar{K}^{0}}=1 / 4$, reflecting the ratio $Q_{[b u]}^{2} / Q_{[b d]}^{2}$ with $Q_{[b u]}=1 / 3$ and $Q_{[b d]}=-2 / 3$ being the effective electric charges for the constituent diquarks of the flavor eigenstates. The predicted spectra in Figure 3 show a distinct resonant behavior and are clearly distinguishable from models like the multipole expansion. We hope, that Belle can improve their current analysis and provide sufficient statistics to show the predicted spectra.

Another way to scrutinize $Y_{b}(10890)$ is via hadroproduction. Finally, the bottom exotica should show in hadronic processes for which $X_{c}$ (3872), measured by CDF [35], is a good example. To distinguish the rescattering model from the tetraquark explanation, discussed in Sec. 2, the decay characteristics of $\Upsilon(6 S)$ need to be measured. In the former, the partial width for the

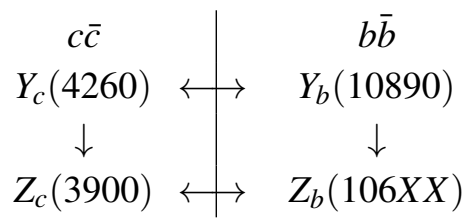

Figure 2: Exotica: Puzzle in the heavy quarks sectors. 

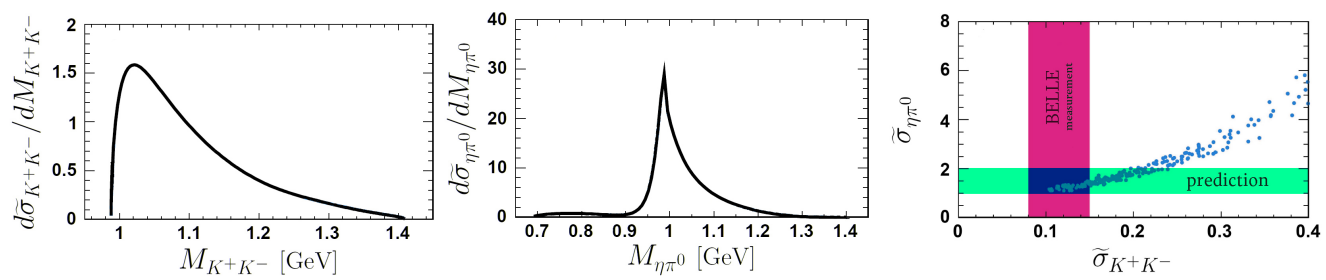

Figure 3: From left to right: Predictions of the $M_{K^{+} K^{-}}$distribution, of the $M_{\eta \pi^{0}}$ distribution and of the correlation between the cross sections of $\sigma_{\Upsilon(1 S) K^{+} K^{-}}$and $\sigma_{\Upsilon(1 S) \eta \pi^{0}}$, normalized by the measured cross section for the $\sigma_{\Upsilon(1 S) \pi^{+} \pi^{-}}$mode. In the two left figures, the dotted (solid) curves show the dimeson invariant mass spectra from the resonant (total) contribution. In the right figure, the dots represent predictions from our fit solutions satisfying $\chi^{2} /$ d.o.f. $<1.6$. The shaded bands shows the current Belle measurement $\widetilde{\sigma}_{K^{+} K^{-}}=0.11_{-0.03}^{+0.04}[15]$ and the prediction for $\sigma_{\Upsilon(1 S) \eta \pi^{0}}$ (the tilde indicates normalization to $\sigma_{\Upsilon(1 S) \pi^{+} \pi^{-}}$).

$\Upsilon(5 S, 6 S) \rightarrow \Upsilon(1 S, 2 S, 3 S) \pi^{+} \pi^{-}$decays are predicted to be roughly of the same order [20]. The (non-)observation of such a pattern will finally refute (or confirm) one of the few exotic candidates in the bottom sector. The theoretical work, based on NRQCD, to enable the experimental analysis is currently in preparation [36].

\section{Conclusion}

The theoretical interpretation to the exotic spectroscopy is inconclusive and a big picture to accommodate all exotic states is missing. A simultaneous explanation of more than just a few states poses a hurdle for all models currently discussed. However, eventually consistent patterns need to emerge and be explained, of which one, pictured in Figure 2, was discussed here. The completion of the list of bottom exotica, but also a confirmation of $Y_{b}(10890)$, discussed in Sec. 6, will be a major step in the direction of testing the models and provide theorists with vital input to present a credible explanation of this new form of QCD.

\section{References}

[1] A. Zupanc [Belle Collaboration], arXiv:0910.3404 [hep-ex].

[2] M. Ablikim et al. [ BESIII Collaboration], arXiv:1303.5949 [hep-ex].

[3] A. Bondar et al. [Belle Collaboration], Phys. Rev. Lett. 108, 122001 (2012) [arXiv:1110.2251 [hep-ex]].

[4] N. A. Tornqvist, Phys. Lett. B 590, 209 (2004) [hep-ph/0402237].

[5] X. Liu, X. -Q. Zeng and X. -Q. Li, Phys. Rev. D 72, 054023 (2005) [hep-ph/0507177].

[6] J. L. Rosner, Phys. Rev. D 76, 114002 (2007) [arXiv:0708.3496 [hep-ph]].

[7] L. Maiani, F. Piccinini, A. D. Polosa and V. Riquer, Phys. Rev. D 71, 014028 (2005) [hep-ph/0412098].

[8] L. Maiani, V. Riquer, F. Piccinini and A. D. Polosa, Phys. Rev. D 72, 031502 (2005) [hep-ph/0507062]. 
[9] N. V. Drenska, R. Faccini and A. D. Polosa, Phys. Lett. B 669, 160 (2008) [arXiv:0807.0593 [hep-ph]].

[10] E. Kou and O. Pene, Phys. Lett. B 631, 164 (2005) [hep-ph/0507119].

[11] D. Ebert, R. N. Faustov and V. O. Galkin, Phys. Lett. B 634, 214 (2006) [hep-ph/0512230].

[12] D. Ebert, R. N. Faustov, V. O. Galkin and W. Lucha, Phys. Rev. D 76, 114015 (2007) [arXiv:0706.3853 [hep-ph]].

[13] N. V. Drenska, R. Faccini and A. D. Polosa, Phys. Rev. D 79, 077502 (2009) [arXiv:0902.2803 [hep-ph]].

[14] J. Beringer et al. [Particle Data Group Collaboration], Phys. Rev. D 86, 010001 (2012).

[15] K. F. Chen et al. [Belle Collaboration], Phys. Rev. Lett. 100, 112001 (2008) [arXiv:0710.2577 [hep-ex]].

[16] I. Adachi et al. [Belle Collaboration], arXiv:0808.2445 [hep-ex].

[17] L. S. Brown and R. N. Cahn, Phys. Rev. Lett. 35, 1 (1975).

[18] A. Ali, C. Hambrock and M. J. Aslam, Phys. Rev. Lett. 104, 162001 (2010) [Erratum-ibid. 107, 049903 (2011)] [arXiv:0912.5016 [hep-ph]].

[19] A. Ali, C. Hambrock and S. Mishima, Phys. Rev. Lett. 106, 092002 (2011) [arXiv:1011.4856 [hep-ph]].

[20] C. Meng and K. -T. Chao, Phys. Rev. D 77, 074003 (2008) [arXiv:0712.3595 [hep-ph]].

[21] K. Nakamura et al. [Particle Data Group Collaboration], J. Phys. G 37, 075021 (2010).

[22] A. E. Bondar, A. Garmash, A. I. Milstein, R. Mizuk and M. B. Voloshin, Phys. Rev. D 84, 054010 (2011) [arXiv:1105.4473 [hep-ph]].

[23] M. B. Voloshin, Phys. Rev. D 84, 031502 (2011) [arXiv:1105.5829 [hep-ph]].

[24] J. -R. Zhang, M. Zhong and M. -Q. Huang, Phys. Lett. B 704, 312 (2011) [arXiv:1105.5472 [hep-ph]].

[25] Y. Yang, J. Ping, C. Deng and H. -S. Zong, J. Phys. G 39, 105001 (2012) [arXiv:1105.5935 [hep-ph]].

[26] Z. -F. Sun, J. He, X. Liu, Z. -G. Luo and S. -L. Zhu, Phys. Rev. D 84, 054002 (2011) [arXiv:1106.2968 [hep-ph]].

[27] M. Cleven, F. -K. Guo, C. Hanhart and U. -G. Meissner, Eur. Phys. J. A 47, 120 (2011) [arXiv:1107.0254 [hep-ph]].

[28] T. Mehen and J. W. Powell, Phys. Rev. D 84, 114013 (2011) [arXiv:1109.3479 [hep-ph]].

[29] J. Nieves and M. P. Valderrama, Phys. Rev. D 84, 056015 (2011) [arXiv:1106.0600 [hep-ph]].

[30] A. Ali, C. Hambrock, I. Ahmed and M. J. Aslam, Phys. Lett. B 684, 28 (2010) [arXiv:0911.2787 [hep-ph]].

[31] A. Ali, C. Hambrock and W. Wang, Phys. Rev. D 85, 054011 (2012) [arXiv:1110.1333 [hep-ph]].

[32] [Belle Collaboration], arXiv:1303.0101 [hep-ex].

[33] B. Aubert et al. [BaBar Collaboration], Phys. Rev. Lett. 95, 142001 (2005) [hep-ex/0506081].

[34] R. Faccini, L. Maiani, F. Piccinini, A. Pilloni, A. D. Polosa and V. Riquer, arXiv:1303.6857 [hep-ph].

[35] V. Khachatryan et al. [CMS Collaboration], Phys. Rev. D 83, 112004 (2011) [arXiv:1012.5545 [hep-ex]].

[36] A. Ali, C. Hambrock, W. Wang, in preparation. 